\title{
Computing quantum eigenvalues made easy
}

\author{
H J Korsch and M Glück \\ FB Physik, Universität Kaiserslautern, D-67653 Kaiserslautern, Germany
}

\begin{abstract}
An extremely simple and convenient method is presented for computing eigenvalues in quantum mechanics by representing position and momentum operators in a simple matrix form. The simplicity and success of the method is illustrated by numerical results concerning eigenvalues of bound systems and resonances for hermitian and non-hermitian Hamiltonians as well as driven quantum systems.
\end{abstract}

Submitted to: J. Phys. A: Math. Gen.

\section{Introduction}

The calculation of eigenvalues is one of the basic problems of elementary quantum mechanics. Consequently numerous techniques have been suggested and used for this purpose and we will not even try to given an overview here. Basis set expansions are certainly very popular. Here the states of the system under consideration are expanded into an adequately chosen basis set $|\nu\rangle, \nu=0,1, \ldots-$ as well as the operators - and the problem is transformed into a matrix problem. Though simple and straightforward in principle, any application requires first the determination of numerical values of the matrixelements. Let us consider here as a typical example the determination of the eigenvalues of the one-dimensional Hamiltonian

$$
H=\frac{p^{2}}{2 M}+V(x),
$$

where the potential $V(x)$ supports bound states. Here one has to compute the matrixelements of the potential,

$$
V_{\nu \mu}=\langle\nu|V| \mu\rangle=\int \varphi_{\nu}^{*}(x) V(x) \varphi_{\mu}(x) \mathrm{d} x
$$

and of the kinetic energy $p^{2} / 2 M$, preferably in closed form. This is only possible for special basis functions and special potentials $V(x)$. A frequent choice is an expansion into harmonic oscillator states. In this case, the matrixelements (2) can be evaluated in closed form, e.g., for $V(x)=x^{k}$, however the resulting formula [1] is not really simple and, moreover, not very easy to derive, which limits its use in elementary applications required in teaching quantum mechanics. Nevertheless, in this way polynomial or Taylor-series potentials can be attacked (see also [2] for a recent discussion of techniques for treating power series potentials). 


\section{Computational recipe}

In the present article we will draw the attention of the readers to an almost equivalent technique, which circumvents the problems mentioned above. The method is very intuitive, easy to understand and to program. Certainly, the method is not new and, hopefully, also used by many others. However, to our surprise we discovered that the method is not known to several colleagues working in this field. Because we could not find a documentation in the literature, this article tries to fill this gap by presenting a short description of the technique and its numerical implementation.

In almost all introductions to quantum mechanics a matrix representation of the position and momentum operators $x$ and $p$ in terms of the normalized eigenstates $|\nu\rangle$ of a harmonic oscillator $H_{0}=p^{2} / 2 M+\omega_{0}^{2} x^{2} / 2$ is derived. Explicitly, the matrices $x_{\nu \mu}=\langle\nu|x| \mu\rangle$ and $p_{\nu \mu}=\langle\nu|p| \mu\rangle \operatorname{read}$

$$
x=\frac{x_{0}}{\sqrt{2}}\left(\begin{array}{ccccc}
0 & \sqrt{1} & 0 & 0 & \ldots \\
\sqrt{1} & 0 & \sqrt{2} & 0 & \ldots \\
0 & \sqrt{2} & 0 & \sqrt{3} & \ldots \\
0 & 0 & \sqrt{3} & 0 & \ldots \\
\vdots & \vdots & \vdots & \vdots & \ddots
\end{array}\right), p=\frac{\mathrm{i} p_{0}}{\sqrt{2}}\left(\begin{array}{ccccc}
0 & -\sqrt{1} & 0 & 0 & \ldots \\
\sqrt{1} & 0 & -\sqrt{2} & 0 & \ldots \\
0 & \sqrt{2} & 0 & -\sqrt{3} & \ldots \\
0 & 0 & \sqrt{3} & 0 & \ldots \\
\vdots & \vdots & \vdots & \vdots & \ddots
\end{array}\right)
$$

where $x_{0}=\left(\hbar / M \omega_{0}\right)^{1 / 2}=\hbar^{1 / 2} s$ and $p_{0}=\left(M \omega_{0} \hbar\right)^{1 / 2}=\hbar^{1 / 2} / s$ with a scaling parameter $s=\left(M \omega_{0}\right)^{-1 / 2}$. In the following, we use units with $M=1, \hbar=1$ and choose the frequency of the harmonic reference Hamiltonian $H_{0}$ as $\omega_{0}=1$, i.e. a scaling parameter $s=1$ in most cases. Other choices are possible depending on the system to be studied.

The calculation of the matrix respresentation of other operators such as the Hamiltonian is straightforward: all we have to do is to replace the operators $x$ and $p$ in the Hamiltonian by the matrices (3). In a numerical application the matrices (3) are truncated at $n=N$, and the truncated $N \times N$-matrices are used in the calculation. As always, such a truncation can cause numerical errors in the results and the convergence of the quantities of interest must be checked.

\subsection{Example 1: Bound states}

In the following examples we consider the Hamiltonian (1). Short programs written in MATLAB, a widespread computation package which allows a very simple coding of matrix operations, clarify the numerical implementation. The program code

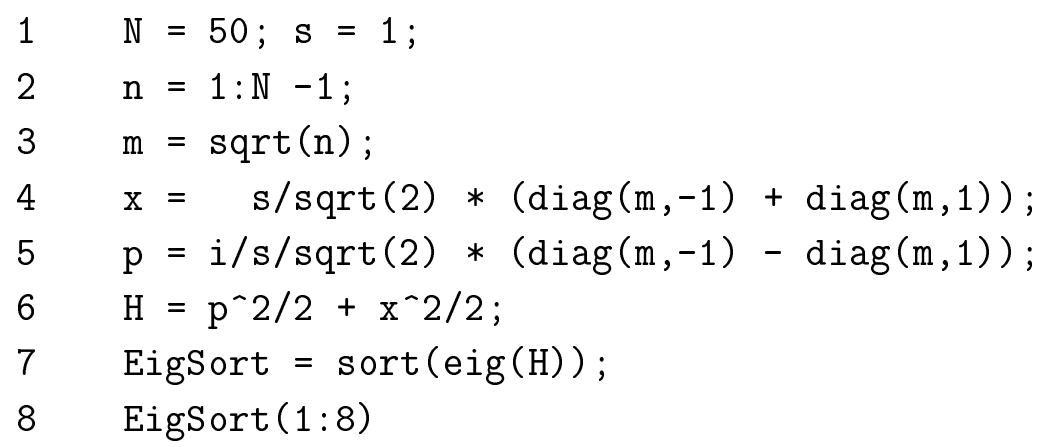


computes the the first $N=50$ eigenvalues of the harmonic oscillator potential $V(x)=x^{2} / 2$. In lines 4 and 5 the matrices (3) are initialized. The Hamiltonian matrix is calculated in line 6. Its eigenvalues are computed in line 7 and directly ordered in increasing magnitude. Note, that the upper eigenvalues may be in error because of the truncation of the basis. The lowest eight eigenvalues are displayed in line 8 . In the trivial harmonic case coded in the program, the matrix $H$ is already diagonal and the results are exact, as expected (note, however, that this is not the case for $s \neq 1$ ).

In order to calculate the eigenvalues for a different potential, one simply changes line 6 of the program. With

$$
6 \quad \mathrm{H}=\mathrm{p}^{\wedge} 2 / 2+\mathrm{x}^{\wedge} 4 / 2 ;
$$

one obtains the eigenvalues for the quartic oscillator $V(x)=x^{4} / 2$. As an example, some numerical results for the lowest seven eigenvalues are listed in Table 1 for several values of the truncation number $N$. Figure 1 illustrates the convergence graphically. We observe a fast convergence towards the exact eigenvalues $E_{n}$ for $N>E_{n}+5$. (The numerical effort, i.e. the computation time increases roughly as $N^{5 / 2}$.)

To some extent, the convergence can be improved by optimizing the scaling parameter $s$, i.e. the width of the harmonic reference Hamiltonian $H_{0}$. Table 2 shows the resulting eigenvalues for $N=15$ for different value of $s$. The scaling parameter $s=0.6$ yields the best numerical performance.

The program can easily be extended to additionally compute the eigenvectors $c_{n}$. Replacing line 7 in the short program example by

$7 \quad[\mathrm{C}, \mathrm{Eig}]=\operatorname{eig}(\mathrm{H})$;

yields the eigenvectors as the columns of the matrix $\mathrm{C}$ (the matrices fulfil $\mathrm{H} * \mathrm{C}=\mathrm{C} * \mathrm{E}$ ig , where Eig is a diagonal matrix). It is then possible to extract the wavefunctions in coordinate space by means of

$$
\psi_{n}(x)=\sum_{\nu=0}^{N} c_{\nu}^{(n)} \varphi_{\nu}(x), \quad \varphi_{n}(x)=\left(\sqrt{\pi} x_{0} 2^{\nu} \nu !\right)^{-1 / 2} \mathrm{e}^{-x^{2} / 2 x_{0}^{2}} H_{\nu}\left(x / x_{0}\right)
$$

where $c_{\nu}^{(n)}$ are the components of the computed eigenvector $c_{n}$ and $\varphi_{\nu}(x)=\langle x \mid \nu\rangle$ is the harmonic oscillator wavefunction. (Note that here and in the following equation $x$ and $p$ are used as variables and not operators.) In the same way

$$
\Psi_{n}(p)=\sum_{\nu=0}^{N} c_{\nu}^{(n)} \Phi_{\nu}(p), \quad \Phi_{n}(p)=\mathrm{i}^{\nu}\left(\sqrt{\pi} p_{0} 2^{\nu} \nu !\right)^{-1 / 2} \mathrm{e}^{-p^{2} / 2 p_{0}^{2}} H_{\nu}\left(p / p_{0}\right)(5)
$$

with $\Phi_{\nu}(p)=\langle p \mid \nu\rangle$ yields the less well-known wavefunctions in momentum space (more on momentum space distributions can be found in $[3,4])$.

Besides, the knowledge of the eigenvectors provides a direct method to calculate expectation values of the eigenstates $|n\rangle$ of $H$, e.g. the potential energy for the $x^{4} / 2-$ potential,

$$
E_{\mathrm{pot}}=\langle n|V| n\rangle=c_{n}^{t} \frac{1}{2} x^{4} c_{n}
$$


In fact, expectation values are the diagonal elements of a matrix representation in the basis of the eigenstates $|n\rangle$. The transformation between both basis sets is governed by the eigenvector matrix $\mathrm{C}$, therefore the program lines

$7 \quad[\mathrm{C}, \mathrm{Eig}]=\operatorname{eig}(\mathrm{H})$;

8 Epot $=C^{\prime} *\left(x^{\wedge} 4 / 2\right) * C$;

(the $\mathrm{C}^{\prime}$ is the adjoint of the matrix $\mathrm{C}$ ) yield the desired matrix $\langle m|V| n\rangle$.

In the same way as demonstrated for the quartic oscillator one can treat all polynomial potentials. For example, the Hamiltonian $\mathrm{H}=\mathrm{p}^{\wedge} 2 / 2+\mathrm{x}^{\wedge} 4 / 4-\mathrm{x}^{\wedge} 2 / 2$ yields the eigenvalues of the double minimum potential $V(x)=x^{4} / 4-x^{2} / 2$. For other cases, the function $V(x)$ of the operator $x$ must be evaluated by, e.g., a Taylor expansion. For the exponential potential this calculation is provided by the MATLAB function expm, so that as another test case one can consider the Morse oscillator

$$
V(x)=D\left(1-\mathrm{e}^{-\beta x}\right)^{2} .
$$

If one replaces the program line defining the Hamiltonian by the modified lines

$6 \mathrm{a} \quad$ One $=$ eye $(\mathrm{N}) ; \mathrm{D}=1 ;$ beta $=0.3$;

$6 \mathrm{~b} \quad \mathrm{H}=\mathrm{p}^{\wedge} 2 / 2+\mathrm{D} *(\text { One }-\operatorname{expm}(-\operatorname{beta} * \mathrm{x}))^{\wedge} 2$;

(note, that the constant 1 in the potential must be replaced by a $N \times N$ unit matrix One=eye $(\mathbb{N}))$, the program yields numerical eigenvalues in agreement with the exact result

$$
E_{n}=\hbar \omega_{0}(n+1 / 2)-\frac{\hbar^{2} \omega_{0}^{2}}{4 D}(n+1 / 2)^{2}, \quad \omega_{0}^{2}=2 D \beta^{2} / M,
$$

where $\omega_{0}$ is the frequency at the bottom of the potential well.

Somewhat more demanding are non-analytic potentials, as for instance the linear potential well $V(x)=|x| / 2$. Here the MATLAB routine funm( $\mathrm{x}$, ' $\mathrm{f}(\mathrm{x})^{\prime}$ ') can be used for a (not so fast) evaluation of the matrix function $f(x)$ : With

$6 \mathrm{H}=\mathrm{p}^{\wedge} 2 / 2+\operatorname{funm}\left(\mathrm{x},{ }^{\prime} \mathrm{abs}^{\prime}\right) / 2 ;$

one can calculate the eigenvalues (see also [5]) for this potential.

\subsection{Example 2: Resonances}

The same program code can also be used for a calculation of resonances. For instance, the model potential

$$
H=\frac{p^{2}}{2}+\left(\frac{x^{2}}{2}-J\right) \mathrm{e}^{-\lambda x^{2}}+J
$$

is considered in many studies as a typical example. This potential is approximately harmonic for small $x$, it increases up to a maximal value and approaches the value $J$ at infinity. The command lines

$6 \mathrm{a} \quad$ One $=$ eye $(\mathrm{N}) ; \mathrm{J}=0.8 ;$ lambda $=0.1 ;$

$6 \mathrm{~b} \quad \mathrm{H}=\mathrm{p}^{\wedge} 2 / 2+\left(\mathrm{x}^{\wedge} 2 / 2-\mathrm{J} * 0\right.$ ne $) * \operatorname{expm}\left(-\right.$ lambda $\left.* \mathrm{x}^{\wedge} 2\right)+\mathrm{J} *$ One 
allow a treatment of this potential. The spectrum for energies $E>J$ is, of course, continuous. However, the potential supports resonances, which manifest themselves, e.g. by a box-quantization. Putting the system into the box $|x|<L$ and increasing the box size $L$ yields real eigenvalues, which undergo a series of avoided crossing at the resonance energies. We can simulate this behavior here by increasing the matrix dimension $N$, i.e. we increase the potential range covered by our harmonic oscillator basis. As an example, Figure 2 shows such a stabilization diagram for the case $J=0.8$, $\lambda=0.1$. The potential supports a bound state at $E_{0} \approx 0.502$ and the most stable resonances appear at $E_{1} \approx 1.421$ and $E_{2} \approx 2.127$ (see [6]; more exact values are listed Table 3). These resonance energies are only slightly affected by a parameter variation and can be read off from the stabilization diagram 2, as well as the width of the resonances by some more well established methods $[7,8]$.

\subsection{Example 3: Non-hermitian Hamiltonians}

It is, of course, not required for the present simple numerical program that the Hamiltonian is hermitian. Eigenvalue problems for non-hermitian Hamiltonians arise quite often (see, e.g. the recent articles $[9,10]$ ). As an example we consider here the case

$$
H=p^{2}+x^{2}(\mathrm{i} x)^{\epsilon}, \quad \epsilon \text { real. }
$$

This Hamiltonian is $\mathcal{P} \mathcal{T}$-symmetric, i.e. invariant under the combined parity, $\mathcal{P}$ : $(x, p) \rightarrow(-x,-p)$, and time-reversal, $\mathcal{T}:(x, p, \mathrm{i}) \rightarrow(x,-p,-\mathrm{i})$, operation (for more details see $[9,10])$. Numerical results obtained by the present method using

$6 \mathrm{a}$ eps $=-0.5$;

$6 \mathrm{~b} \quad \mathrm{H}=\mathrm{p}^{\wedge} 2+\mathrm{x}^{\wedge} 2 *(i * \mathrm{x})^{\wedge}$ eps ;

for $\epsilon=1$ and $\epsilon=-0.5$ are listed in Table 3. For $\epsilon=1$ the eigenvalues are real and the eigenvectors are $\mathcal{P} \mathcal{T}$-symmetric, whereas and for $\epsilon=-0.5$ only the lowest eigenvalues are real with $\mathcal{P} \mathcal{T}$-symmetric eigenstates, whereas the higher ones split into pairs of complex conjugate eigenvalues and the $\mathcal{P} \mathcal{T}$-symmetry of the eigenstates is broken. In the numerical calculations a scaling parameter $s=0.6$ has been used.

Another example of a non-hermitian Hamiltonian arises if one uses complex-scaling methods [11] for calculating resonance states as square integrable eigenstates of a complex-scaled Hamiltonian. In its most simple version, this is just a complex rotation of the coordinate

$$
x \longrightarrow x \mathrm{e}^{\mathrm{i} \theta}, \quad p \longrightarrow p \mathrm{e}^{-\mathrm{i} \theta},
$$

provided that the rotation angle is chosen reasonably. Using our MATLAB program, one can simply use a complex scaling parameter $s$. Replacing the corresponding lines of the program by

$1 \quad \mathrm{~N}=60 ;$ theta $=0.75 ; \mathrm{s}=\exp (i *$ theta $)$;

$6 \mathrm{a} \quad$ One $=$ eye $(\mathrm{N}) ; \mathrm{J}=0.8 ;$ lambda $=0.1$;

$6 \mathrm{~b} \quad \mathrm{H}=\mathrm{p}^{-} 2 / 2+\left(\mathrm{x}^{-} 2 / 2-\mathrm{J} *\right.$ One $) * \operatorname{expm}\left(-\operatorname{lambda} * \mathrm{x}^{-} 2\right)+\mathrm{J} *$ One 
it computes the resonance energies in the potential (9). Table 3 shows the complex resonance energy $\mathcal{E}=E-\mathrm{i} \Gamma / 2$ computed with these parameter settings.

\subsection{Example 4: Driven quantum systems}

As a last example we will discuss Hamiltonians which depend explicitly on time, modeling for example systems in external time-dependent fields (see the review article by Grifoni and Hänggi [12] or the textbooks by Dittrich at al. [13], Chapter 5, or by Bayfield [14]). In particular, we consider time-periodic systems with period $T$. Here it is convenient to analyze the dynamics in terms of the so-called Floquet states [12-14], which are the eigenstates of the time evolution operator $U(t, 0)$ over one period, the so-called Floquet operator. These Floquet states can be used essentially in the same manner as the eigenstates of a time-independent Hamiltonian. Because of unitarity, the eigenvalues are of unit magnitude and can be conveniently written as $\exp \left(-\mathrm{i} E_{\alpha} T / \hbar\right)$, where the so-called quasienergies $E_{\alpha}$ are defined modulo integer multiples of $\hbar \omega$, i.e. we have a family of quasienergies

$$
E_{\alpha}=E_{n, \ell}=E_{n, 0}+\ell \hbar \omega, \quad n=0,1,2, \ldots, \quad \ell=0, \pm 1, \pm 2, \ldots .
$$

Numerically, the time-evolution operator $U=U(T, 0)$ can be calculated by splitting the time period $T$ into $J$ equidistant intervals and approximating the time-dependent Hamiltonian $H(t)$ in the $j$-th interval by the value $H\left(t_{j}\right)$ with $t_{j}=j T / J, j=1,2, \ldots, J$. Then the Floquet operator is given by the product

$$
U(T, 0)=\mathrm{e}^{-\mathrm{i} H\left(t_{J}\right) \delta / \hbar} \cdots \mathrm{e}^{-\mathrm{i} H\left(t_{2}\right) \delta / \hbar} \mathrm{e}^{-\mathrm{i} H\left(t_{1}\right) \delta / \hbar},
$$

where $\delta=T / J$ is the time-step and $J$ is chosen sufficiently large. After this time propagation, the eigenvalues $\lambda=\mathrm{e}^{-\mathrm{i} E T / \hbar}$ of the resulting matrix $U$ are computed. Finally, $\mathrm{i} \hbar \log (\lambda) / T$ yields the desired quasienergies.

As a first demonstration, we consider the celebrated forced harmonic oscillator, which is one of the rare cases that allow a closed form solution. In particular we choose a time-periodic harmonic driving, i.e.

$$
H(t)=\frac{p^{2}}{2}+\frac{\omega_{0}^{2}}{2} x^{2}+f x \cos \omega t=H_{0}+f x \cos \omega t .
$$

with period $T=2 \pi / \omega$. For this Hamiltonian, the exact quasienergies are, up to multiples of $\hbar \omega$, given by (see, e.g. [13])

$$
E_{n}=\hbar \omega_{0}(n+1 / 2)+\frac{f^{2}}{4\left(\omega^{2}-\omega_{0}^{2}\right)} .
$$

In fact, one observes just the harmonic oscillator ladder of $H_{0}$ shifted by a stateindependent dynamical Stark shift.

The following MATLAB program lines first calculate the Floquet operator and its eigenvalues and eigenstates, which are then ordered according to the expectation values of $H_{0}$. Such an ordering is necessary in order to number and identify the states, because the Floquet eigenvalues have no intrinsic order in contrast to the eigenvalues of a timeindependent Hamiltonian. Units are chosen as $\omega_{0}=1, \hbar=1$ (the missing lines are identical to the original program lines). 


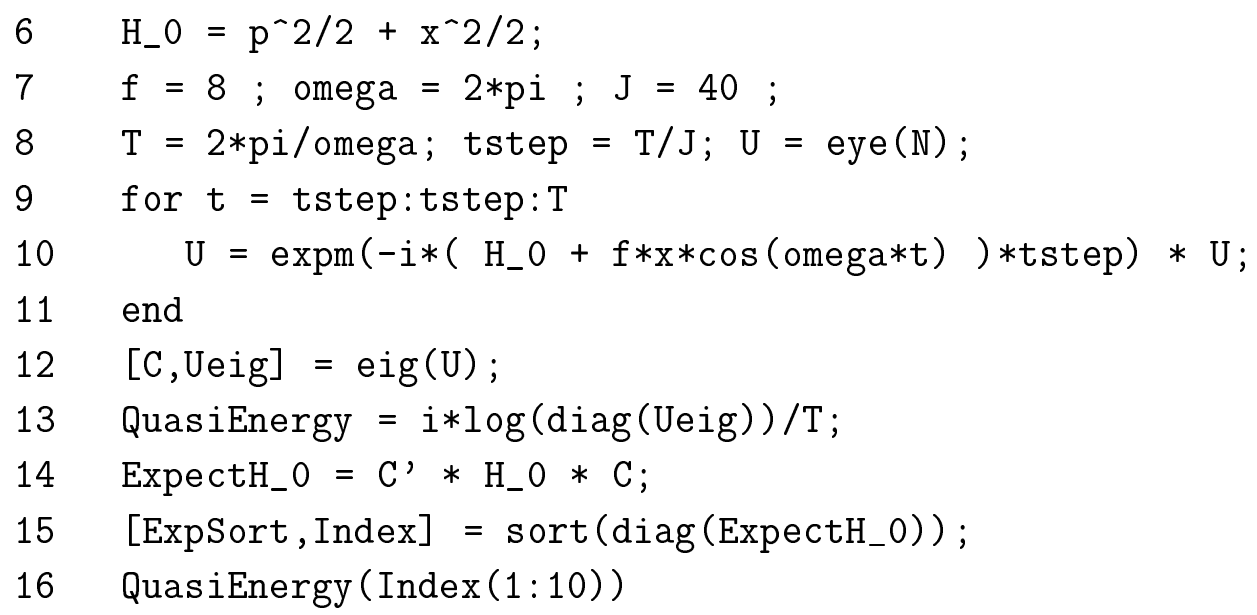

(The vector Index computed in line 15 contains the positions of the sorted expectation values on the diagonal of the matrix ExpectH_0.).

In Table 4 we list the resulting quasienergies reduced to the interval $-\hbar \omega<E_{n}<$ $+\hbar \omega$, the first Brillouin zone, for parameters $\omega=2 \pi, f=8 ; N=30$ is the matrix size and $s=1$ the scaling factor. Results for $J=10,20,40$ are listed, which approach the exact values $(15)$.

In the same way, more interesting systems can be studied without elaborate programming or computational effort. To show this, we will consider a study of an excitation of an anharmonic oscillator, modelling a vibrational excitation of an $\mathrm{HF}-$ molecule in a strong Laser field. The system considered by Holthaus and Just [15] for this purpose is a forced Morse oscillator (see (7)):

$$
H(t)=\frac{p^{2}}{2 M}+D\left(1-\mathrm{e}^{-\beta x}\right)^{2}+d x f \cos \omega t
$$

with parameters $\hbar=1, M=1744.8, D=0.2251, \beta=1.174, d=0.3099$.

First, we test our numerical method for this case by computing numerically the eigenvalues of the field-free Hamiltonian as described above, using the scaling parameter $s=\left(M \omega_{0}\right)^{-1 / 2}$, where $\omega_{0}$ is given in (8). With, e.g., $N=25$ one obtaines $E_{0}=0.009330, E_{1}=0.027398, E_{2}=0.044677, E_{3}=0.061165, E_{4}=0.076863, E_{5}=$ 0.091772 in agreement with the exact formula (8). As in [15] we will tune the Laser frequency to the $0 \rightarrow 5$ transition and choose

$$
\omega=\left(E_{5}-E_{0}\right) / 5 \hbar=0.016488,
$$

i. e. a five-photon resonance. For a given field amplitude $f$ the quasienergy spectrum can be calculated in the same manner as described above for the forced harmonic oscillator, where the field-free Hamiltonian is changed to

$\mathrm{H}_{-} \mathrm{O}=\mathrm{p}^{\wedge} 2 / 2 / \mathrm{M}+\mathrm{D} *(\text { One }-\operatorname{expm}(-\operatorname{beta} * \mathrm{x}))^{\wedge} 2$;

An understanding of the dynamical behavior, however, can be obtained from a knowledge ot the quasienergies as a function of the field amplitude $f$. This dependence is calculated and plotted by the following MATLAB program: 
$1 \quad \mathrm{~N}=30 ;$ One $=$ eye $(\mathrm{N})$;

$2 \quad \mathrm{M}=1744.8 ; \mathrm{D}=0.2251 ;$ beta $=1.174 ; \mathrm{d}=0.3099 ;$ omega $=0.016488 ;$

3 omega_ $0=\operatorname{sqrt}\left(2 * D *\right.$ beta $\left.^{\wedge} 2 / M\right) ; s=(M * \text { omega_0 })^{\wedge}-0.5$;

$4 \mathrm{n}=1: \mathrm{N}-1$;

$5 \mathrm{~m}=\operatorname{sqrt}(\mathrm{n})$;

$6 \mathrm{x}=\mathrm{s} / \operatorname{sqrt}(2) *(\operatorname{diag}(\mathrm{m},-1)+\operatorname{diag}(\mathrm{m}, 1)) ;$

$7 \mathrm{p}=\mathrm{i} / \mathrm{s} / \operatorname{sqrt}(2) *(\operatorname{diag}(\mathrm{m},-1)-\operatorname{diag}(\mathrm{m}, 1)) ;$

$8 \quad \mathrm{H}_{-} \mathrm{O}=\mathrm{p}^{\wedge} 2 / 2 / \mathrm{M}+\mathrm{D} *(\text { One }-\operatorname{expm}(-\operatorname{beta} * \mathrm{x}))^{\wedge} 2$;

9 for $\mathrm{nf}=0: 100$

$10 \mathrm{f}=\mathrm{nf} * 0.001$

$11 \mathrm{~J}=60 ; \mathrm{T}=2 * \mathrm{pi} /$ omega; tstep $=\mathrm{T} / \mathrm{J}$;

$12 \mathrm{U}=\operatorname{eye}(\mathrm{N})$;

13 for $\mathrm{t}=$ tstep:tstep: $\mathrm{T}$

$14 \mathrm{U}=\operatorname{expm}\left(-\mathrm{i} *\left(\mathrm{H}_{-} \mathrm{O}+\mathrm{f} * \mathrm{~d} * \mathrm{x} * \cos (\right.\right.$ omega $\left.* t)\right) *$ tstep $) * \mathrm{U}$;

15 end

$16 \quad[$ C,Ueig $]=\operatorname{eig}(\mathrm{U})$;

17 QuasiEnergy $=i * \log (\operatorname{diag}(\operatorname{Ueig})) / \mathrm{T}$;

18 ExpectH_O $=\mathrm{C}^{\prime} * \mathrm{H}_{-} \mathrm{O} * \mathrm{C}$;

19 [ExpSort, Index $]=\operatorname{sort}(\operatorname{diag}($ ExpectH_0 $))$;

$20 \quad \mathrm{QE}(:, \mathrm{nf}+1)=$ QuasiEnergy $(\operatorname{Index}(1: 7))$;

21 end

$22 \mathrm{QE}=\mathrm{QE}+\operatorname{omega*}(\mathrm{QE}<0.2 *$ omega $)$;

$23 \operatorname{plot}(0: 0.001: 0.1, \operatorname{sort}(\mathrm{QE} /$ omega $)) ; \operatorname{axis}\left(\left[\begin{array}{llll}0 & 0.1 & 0.4 & 1.1\end{array}\right]\right)$

Figure 3 shows the resulting plot of the computed quasienergies in units of $\hbar \omega$ for $n=0, \ldots, 5$. In the limit $f \rightarrow 0$ the quasienergies (modulo $\hbar \omega$ ) approach the Morse oscillator energies. Because of the resonant driving, $E_{5,-5} / \hbar \omega$ and $E_{0,0} / \hbar \omega$ are degenerate. The figure reproduces the results presented in [15]. It is, of course, not our intension here to discuss the conclusions which can be drawn from such a plot. Let us just remark that the probability for a $0 \rightarrow 5$ transition in an experiment with a Laser puls with envelope $f=f(t)$ is approximately given by

$$
P_{0 \rightarrow 5}=\sin ^{2}\left(\frac{1}{2 \hbar} \int \mathrm{d} t\left(E_{5}^{f(t)}-E_{0}^{f(t)}\right)\right),
$$

where $E_{n}^{f(t)}$ denotes the instantaneous quasienergies (see [15] for more details).

\section{Concluding remarks}

The few examples presented in this article have demonstrated that a simple and wellknown representation of the position and momentum opertors allow very simple and easily producable computer codes for a study of elementary and more advanced topics in quantum mechanics. Much more can be done, of course, and we hope to stimulate further application of this technique both for teaching physics and for lower level research projects. 


\section{References}

[1] R. M. Wilcox, J. Math. Phys. 8 (1967) 962

[2] J. P. Killingbeck, T. Scott, and B. Rath, J. Phys. A 33 (2000) 6999

[3] H. J. Korsch and B. Schellhaaß, Eur. J. Phys. 21 (2000) 63

[4] H. J. Korsch and B. Schellhaaß, Eur. J. Phys. 21 (2000) 73

[5] H. J. Korsch, Chem. Phys. Lett. 85 (1982) 558

[6] M. Rittby, N. Elander and E. Brändas, Phys. Rev. A 26(3) (1982) 1804

[7] A. U. Hazi and H. S. Taylor, Phys. Rev. A 1 (1970) 1109

[8] C. H. Maier, L. S. Cederbaum, and W. Domcke, J. Phys. B 13 (1980) L119

[9] C. M. Bender and S. Boettcher, Phys. Rev. Lett. 80 (1998) 5243

[10] C. M. Bender, S. Boettcher, and P. N. Meisinger, J. Math. Phys. 40 (1999) 2201

[11] N. Moiseyev, Phys. Rep. 302 (1998) 211

[12] M. Grifoni and P. Hänggi, Phys. Rep. 304 (1998) 229

[13] W. Dittrich and M. Reuter, Classical and Quantum Dynamics, Springer, Berlin, Heidelberg, 1992

[14] J. E. Bayfield, Quantum Evolution, John Wiley and Sons, New York, 1999

[15] M. Holthaus and B. Just, Phys. Rev. A 49 (1994) 1950 
Table 1. Eigenvalues of the quartic oscillator $V(x)=x^{4} / 2$ for various choices of the basis size $N$.

\begin{tabular}{rrrrrrr}
\hline$n$ & \multicolumn{5}{c}{$E_{n}$} \\
\hline & \multicolumn{1}{c}{$N=10$} & \multicolumn{1}{c}{$N=15$} & \multicolumn{1}{c}{$N=20$} & \multicolumn{1}{c}{$N=30$} & \multicolumn{1}{c}{$N=40$} & \multicolumn{1}{c}{ exact } \\
\hline 0 & 0.529804 & 0.530150 & 0.530180 & 0.530181 & 0.530181 & 0.530181 \\
1 & 1.884380 & 1.899519 & 1.899802 & 1.899836 & 1.899837 & 1.899837 \\
2 & 3.703755 & 3.722122 & 3.727699 & 3.727848 & 3.727849 & 3.727849 \\
3 & 5.497667 & 5.829293 & 5.821052 & 5.822354 & 5.822373 & 5.822373 \\
4 & 7.092175 & 8.196389 & 8.137202 & 8.130918 & 8.130912 & 8.130913 \\
5 & 8.274857 & 9.824282 & 10.605741 & 10.619471 & 10.619179 & 10.619186 \\
6 & 22.115362 & 10.387204 & 13.088132 & 13.264731 & 13.264260 & 13.264236 \\
\hline
\end{tabular}

Table 2. Eigenvalues of the quartic oscillator $V(x)=x^{4} / 2$ for various choices of the scaling parameter $s$.

\begin{tabular}{rrrrrrr}
\hline$n$ & \multicolumn{5}{c}{$E_{n}$} \\
\hline & \multicolumn{1}{c}{$s=0.4$} & \multicolumn{1}{c}{$s=0.6$} & \multicolumn{1}{c}{$s=0.8$} & \multicolumn{1}{c}{$s=1.0$} & \multicolumn{1}{c}{$s=1.2$} & \multicolumn{1}{c}{ exact } \\
\hline 0 & 0.527901 & 0.530181 & 0.530181 & 0.530150 & 0.530191 & 0.530181 \\
1 & 1.904213 & 1.899836 & 1.899831 & 1.899519 & 1.903072 & 1.899837 \\
2 & 3.579742 & 3.727846 & 3.727643 & 3.722122 & 3.735836 & 3.727849 \\
3 & 5.914909 & 5.822367 & 5.822170 & 5.829293 & 5.672530 & 5.822373 \\
4 & 6.422003 & 8.130530 & 8.126915 & 8.196389 & 6.770660 & 8.130913 \\
5 & 10.536731 & 10.619243 & 10.629422 & 9.824282 & 11.899233 & 10.619186 \\
6 & 11.310578 & 13.255599 & 12.723456 & 10.387204 & 12.652289 & 13.264236 \\
\hline
\end{tabular}


Table 3. Eigenvalues of non-hermitian Hamiltonians. The eigenvalues for the $\mathcal{P} \mathcal{T}$ symmetric Hamiltonian (10) are real for $\epsilon=1$ (systems $A_{1}$ ) and both real and complex for $\epsilon=-0.5$ (systems $\mathrm{A}_{2}$ ); the complex resonance energies for the potential (9) (system B) are calculated by complex scaling (see text).

\begin{tabular}{crrrrrrl}
\hline$n$ & \multicolumn{2}{c}{$E_{n}\left(\right.$ system $\left.\mathrm{A}_{1}\right)$} & \multicolumn{2}{c}{$E_{n}\left(\right.$ system $\left.\mathrm{A}_{2}\right)$} & $E_{n}$ & (system B) \\
\hline 0 & 1.1563 & $-0.0000 \mathrm{i}$ & 1.0869 & $-0.0000 \mathrm{i}$ & 0.5020 & $-0.0000 \mathrm{i}$ \\
1 & 4.1092 & $-0.0000 \mathrm{i}$ & 3.1958 & $-0.0000 \mathrm{i}$ & 1.4210 & $-5.8 \cdot 10^{-5} \mathrm{i}$ \\
2 & 7.5623 & $-0.0000 \mathrm{i}$ & 4.4221 & $-0.0000 \mathrm{i}$ & 2.1272 & $-0.0154 \mathrm{i}$ \\
3 & 11.3144 & $-0.0000 \mathrm{i}$ & 6.6559 & $-0.9513 \mathrm{i}$ & 2.5846 & $-0.1738 \mathrm{i}$ \\
4 & 15.2916 & $-0.0000 \mathrm{i}$ & 6.6559 & $+0.9513 \mathrm{i}$ & 2.9244 & $-0.5648 \mathrm{i}$ \\
5 & 19.4516 & $-0.0000 \mathrm{i}$ & 9.0912 & $+1.9946 \mathrm{i}$ & 3.2555 & $-1.1115 \mathrm{i}$ \\
6 & 23.7667 & $-0.0000 \mathrm{i}$ & 9.0912 & $-1.9946 \mathrm{i}$ & 3.5572 & $-1.7555 \mathrm{i}$ \\
\hline
\end{tabular}

Table 4. Quasienergies reduced to the first Brillouin zone for the forced harmonic oscillator (14) for $\omega=\hbar=1, \omega=2 \pi$ and $f=8$ for various numbers $J$ of time-steps. Also given are the exact values (15).

\begin{tabular}{rrrrr}
\hline$n$ & \multicolumn{4}{c}{$E_{n}$} \\
\hline & \multicolumn{1}{c}{$J=10$} & \multicolumn{1}{c}{$J=20$} & \multicolumn{1}{l}{$J=40$} & exact \\
\hline 0 & 0.902410 & 0.912414 & 0.914964 & 0.915818 \\
1 & 1.902410 & 1.912414 & 1.914964 & 1.915818 \\
2 & 2.902410 & 2.912414 & 2.914964 & 2.915818 \\
3 & -2.380776 & -2.370771 & -2.368222 & -2.367368 \\
4 & -1.380776 & -1.370771 & -1.368222 & -1.367368 \\
5 & -0.380775 & -0.370771 & -0.368222 & -0.367368 \\
6 & 0.619224 & 0.629229 & 0.631778 & 0.632632 \\
7 & 1.619224 & 1.629229 & 1.631778 & 1.632632 \\
8 & 2.619224 & 2.629229 & 2.631778 & 2.632632 \\
9 & -2.663961 & -2.653956 & -2.651407 & -2.650553 \\
\hline
\end{tabular}




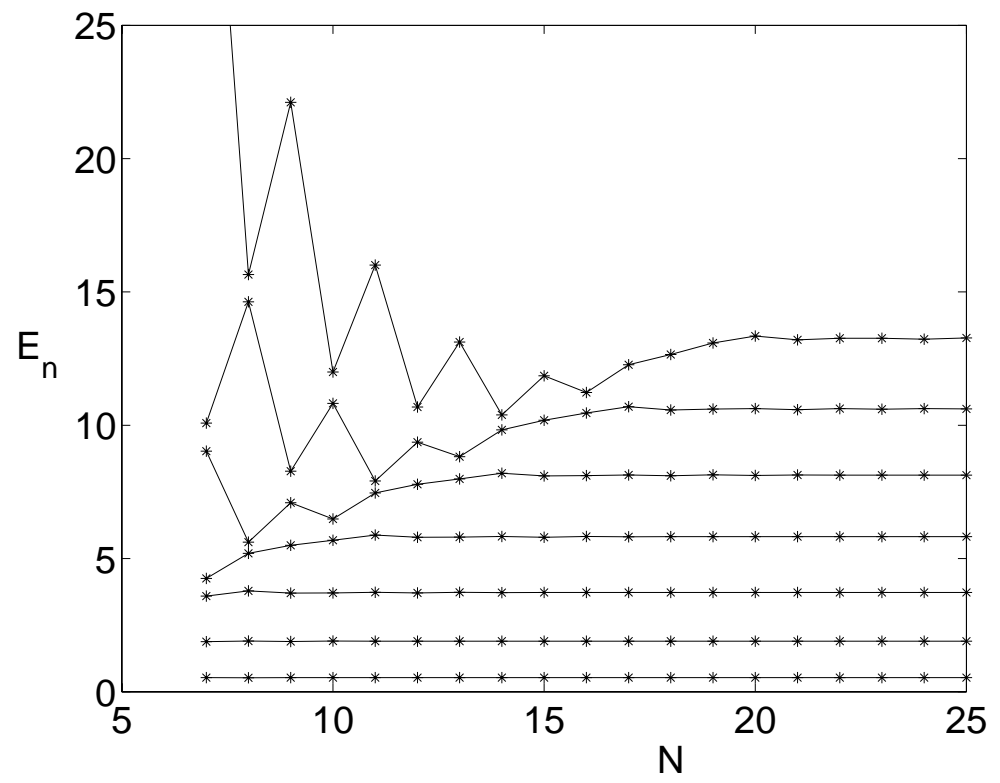

Figure 1. Convergence behavior of the computed eigenvalues for the quartic oscillator $V(x)=x^{4} / 2$ as a function of the matrix size $N$.

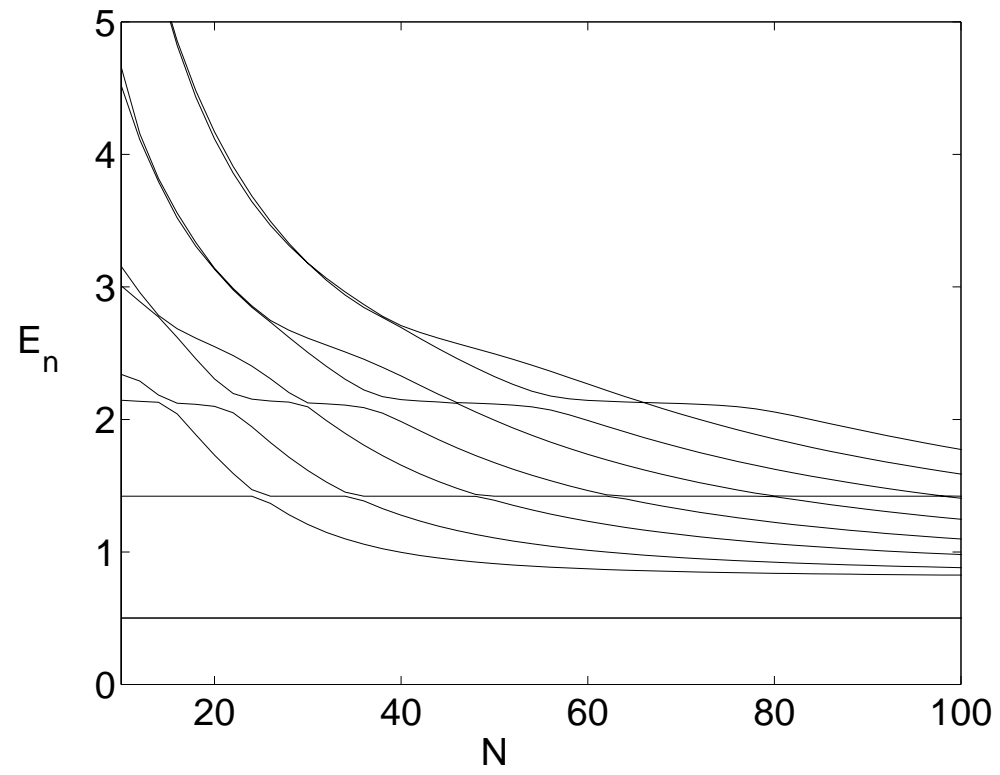

Figure 2. A stabilization diagram, real eigenvalues as a function of the matrix size $N$ reveals the lowest bound state and resonances for potential (9) for potential parameters $J=0.8, \lambda=0.1(s=1)$; only even $N$ are shown. 


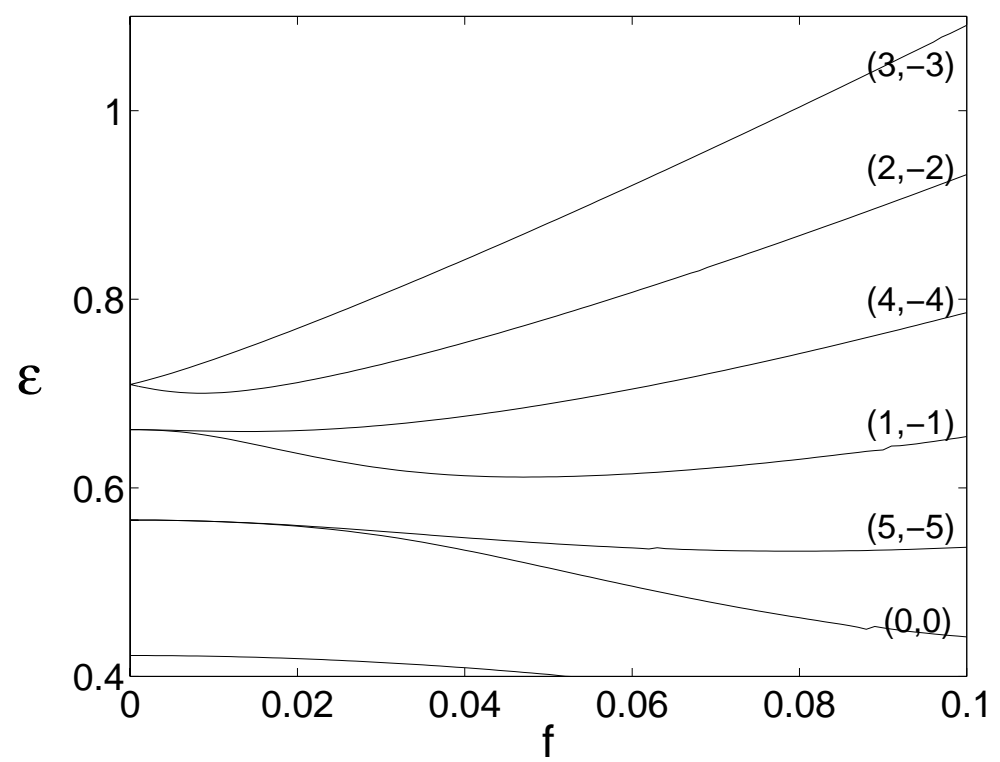

Figure 3. Computed quasienergies $E_{n, \ell}=E_{n, 0}+\ell \hbar \omega$ for the driven Morse oscillator as a function of the driving amplitude $f$. The label $(n, \ell)$ indicates that a quasienergy approaches $E_{n}+\ell \hbar$ for $f \rightarrow 0$. The driving frequency $\omega$ is in resonance with the $n=0 \rightarrow n=5$ transition of the field-free oscillator. Shown is $\epsilon_{n, \ell}=E_{n, \ell} / \hbar \omega$ for $n=0, \ldots 5$. The figure reproduces the results in [15]. 